\title{
Fractal Features of Specific Emitter Identification
}

\author{
J. DUDCZYK ${ }^{a, *}$ AND A. KAWALEC ${ }^{b}$ \\ ${ }^{a}$ WB Electronics S.A., Poznańska 129/133, 05-850 Ożarów Mazowiecki, Poland \\ ${ }^{b}$ Institute of Radioelectronics, Faculty of Electronics, Military University of Technology \\ S. Kaliskiego 2, 00-908 Warsaw, Poland
}

\begin{abstract}
This article presents the issues connected with emitter sources identification with low distinctive primary features of a signal. It is a specific type of identification called specific emitter identification, which distinguishes different copies of the same type of emitter. The term of specific emitter identification was presented on the basis of fractal features received from the transformation of measurement data sets. The use of linear regression and Lagrange polynomial interpolation resulted in the estimation of measurement function. The method analysing properties of measurement function which was suggested by the authors caused the extraction of two additional distinctive features. The features above extended the vector of basic radar signals' parameters. The extended vector of radar signals' features made it possible to identify the copy of emitter source.
\end{abstract}

DOI: 10.12693/APhysPolA.124.406

PACS: 42.30.Sy, 47.54.-r, 43.60.Lq

\section{Introduction}

Signal identification using classic techniques based on the statistic of analysis of basic measurable signal parameters is not sufficient for the specific emitter identification (SEI) problems [1, 2]. A signal with low distinctive primary features is a radar signal with a simple (not complicated) and a stable structure (without modulation inside the impulse, on constant radio-frequency (RF), constant pulse repetition interval (PRI) and pulse width (PW)). Specific identification method of the same type radar copies is the extraction of distinctive features which identify this copy [3].

The distinctive features above may be a result of the received transformations of measurement data sets. New data sets will have fractal features which will make it possible to define clearly the source of emission. The fractal features above and the theory of fractals is adopted by researchers especially in the field of synthetic aperture radar (SAR) image transformation $[4,5]$, acoustic signal transformation and the analysis of radar signals. As the authors of this article claim, the identification of emitter sources based on classical methods of the analysis of basic parameters is currently inefficient. The methods of SEI $[1,6]$ should be used in order to identify a radar copy of the same type more precisely.

The process of identification was done on the basis of the fractal which appeared from the transformation of the so-called measurement points for which the recording of primary features of a radar signal was prepared. Measurement points correspond to further frequency values of which the recording of a signal was done. Given sets of measurement points underwent transformation in the two-dimensional Euclidean plane. As a result of the transformation above the transformation attractor was received. This attractor might be particularly a fractal.

*corresponding author; e-mail: j.dudczyk@wb.com.pl
A set of affine transformations is in this case iterated function system (IFS), and the fractal of generalized measurement function is the attractor of the described radar SEI procedure. The number of measurement points was chosen experimentally and is the double value of maximum width of the indirect frequency filter of the superheterodyne receiver i.e. $40 \mathrm{MHz}$. From this assumption appears the fact that the border primary number of measurement points is not bigger than 80 . As a result of the research, particular collections of values of measurement points were confirmed and were the condition which was sufficient to extract additional distinctive features of the radar emission sources.

2. Transformation fractal of characteristic points

Given set of measurement points was assigned in the form of right-hand measurement vectors $\boldsymbol{p}^{\mathrm{r}}$ and left-hand ones $\boldsymbol{p}^{\mathrm{l}}$ with the beginning in a chosen reference point, so that $\boldsymbol{p}^{\mathrm{r}}=\left[p_{1}^{\mathrm{r}}, p_{2}^{\mathrm{r}}, \ldots, p_{N}^{\mathrm{r}}\right]^{\mathrm{T}}$ and $\boldsymbol{p}^{\mathrm{l}}=\left[p_{1}^{\mathrm{1}}, p_{2}^{\mathrm{l}}, \ldots, p_{M}^{\mathrm{l}}\right]^{\mathrm{T}}$. In order to define the desirable selective features the $T: \boldsymbol{p}^{\mathrm{r}} \rightarrow t$ transformation was done. In this transformation $t$ is the image of the $\boldsymbol{p}^{\mathrm{r}}$ vector in the form of a vector with coordinates corresponding to the $\boldsymbol{p}^{1}$ vector. For the transparent record of the transformation above with the use of vectors: $\boldsymbol{p}^{\mathrm{r}}$ and $\boldsymbol{p}^{\mathrm{l}}$, the above mapping was written in the Euclidean plane, that is $T: E^{1} \rightarrow E^{2}$. In the issue, which is considered here, these transformations are linear mappings, so they can be written in the matrix form as $t=T\left(\boldsymbol{p}^{\mathrm{p}}, \mathrm{A}\right)$, in which $\mathrm{A}$ is the matrix of a given transformation. Depending on the received symmetry or asymmetry (right/left-hand) of measurement points they will create dispersion graphs given by [6]. Measurement points presented in Fig. 1, transformed and depicted together, form the so-called measurement function $K\left(f_{n}\right)$. Figure 1 shows the coordinate plane, where an abscissa (the value of $x$ ) is marked as an $f_{n}^{x}$ and an ordinate (the value of $y$ ) is marked as an $f_{n}^{y}$.

On the basis of distinctive streaks which were formed, such hypothesis can be proposed: functions $g_{A}\left(f_{n}\right)$, $g_{B}\left(f_{n}\right), g_{C}\left(f_{n}\right)$, and $g_{D}\left(f_{n}\right)$ belong to the class of linear functions, in which $g_{A}\left(f_{n}\right), g_{B}\left(f_{n}\right), g_{C}\left(f_{n}\right)$, and 


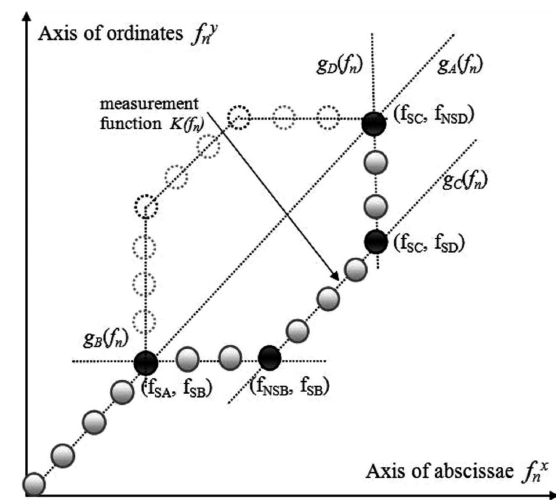

Fig. 1. A graph of measurement points dispersion after transformation in two-dimensional Euclidean plane $E^{2}$ in combined depicting.

$g_{D}\left(f_{n}\right)$ will be the regression lines of the second type for the streaks formed through the measurement points $[7,8]$. Linear equation of regression for the presented case is defined with the following equation: $\boldsymbol{g}\left(f_{n}\right)=$ $\boldsymbol{\alpha} f_{n}+\boldsymbol{\beta}$, in which $\boldsymbol{\alpha}$ can be expressed as a vector $\left[\alpha_{A}, \alpha_{B}, \alpha_{C}, \alpha_{D}\right]^{\mathrm{T}}$ and $\boldsymbol{\beta}$ can be expressed as a vector $\left[\beta_{A}, \beta_{B}, \beta_{C}, \beta_{D}\right]^{\mathrm{T}}$ and $\boldsymbol{g}\left(f_{n}\right)$ can be expressed as a vector $\left[g_{A}\left(f_{n}\right), g_{B}\left(f_{n}\right), g_{C}\left(f_{n}\right), g_{D}\left(f_{n}\right)\right]^{\mathrm{T}}$. To define the value of $\boldsymbol{\alpha}$ and $\boldsymbol{\beta}$ the following equation should be minimized:

$$
\begin{aligned}
& E\left[f_{n}^{Y}-\boldsymbol{\alpha} f_{n}-\boldsymbol{\beta}\right]^{2}=\min , \\
& \left\{\begin{array}{c}
\frac{\partial}{\partial \alpha} E\left[f_{n}^{Y}-\boldsymbol{\alpha} f_{n}-\boldsymbol{\beta}\right]^{2} \\
=-2 E\left[\left(f_{n}^{Y}-\boldsymbol{\alpha} f_{n}-\boldsymbol{\beta}\right) f_{n}\right], \\
\frac{\partial}{\partial \beta} E\left[f_{n}^{Y}-\boldsymbol{\alpha} f_{n}-\boldsymbol{\beta}\right]^{2} \\
=-2 E\left[\left(f_{n}^{Y}-\boldsymbol{\alpha} f_{n}-\boldsymbol{\beta}\right)\right],
\end{array}\right.
\end{aligned}
$$

in which $E$ means expected value. After comparing the calculated derivatives of Eq. (2) to zero, there appears the system of normal equations in which after replacing the expected values with particular moments of equation systems the following relations can be written:

$$
\left\{\begin{array}{c}
\boldsymbol{\alpha} m_{20}+\boldsymbol{\beta} m_{10}=m_{11}, \\
\boldsymbol{\alpha} m_{10}+\boldsymbol{\beta}=m_{01},
\end{array}\right.
$$

in which $m_{10}$ and $m_{01}$ are sample 1 th moments, $m_{20}$ is sample 2 nd moment and $m_{11}$ is mixed sample 1th moment. After further transformations the regression equation is as follows:

$$
g\left(f_{n}\right)=\frac{\mu_{11}}{\mu_{20}} f_{n}+\left(m_{01}-\frac{\mu_{11}}{\mu_{20}} m_{10}\right)=\boldsymbol{\alpha}_{21} f_{n}+\boldsymbol{\beta},
$$

in which

$$
\begin{aligned}
& \boldsymbol{\alpha}_{21}=\left[\frac{\mu_{11}^{A}}{\mu_{20}^{A}}, \frac{\mu_{11}^{B}}{\mu_{20}^{B}}, \frac{\mu_{11}^{C}}{\mu_{20}^{C}}, \frac{\mu_{11}^{D}}{\mu_{20}^{D}}\right]^{\mathrm{T}}=\left[\alpha_{A}, \alpha_{B}, \alpha_{C}, \alpha_{D}\right]^{\mathrm{T}}, \\
& \boldsymbol{\beta}=\left[m_{01}^{A}-\frac{\mu_{11}^{A}}{\mu_{20}^{A}} m_{10}^{A}, m_{01}^{B}-\frac{\mu_{11}^{B}}{\mu_{20}^{B}} m_{10}^{B},\right. \\
& \left.m_{01}^{C}-\frac{\mu_{11}^{C}}{\mu_{20}^{C}} m_{10}^{C}, m_{01}^{D}-\frac{\mu_{11}^{D}}{\mu_{20}^{D}} m_{10}^{D}\right]^{\mathrm{T}} \\
& =\left[\beta_{A}, \beta_{B}, \beta_{C}, \beta_{D}\right]^{\mathrm{T}}
\end{aligned}
$$

and $\mu_{11}$ means mixed 2 nd central moment and $\mu_{20}$ means 2nd central moment. As a result of further transformations four linear regression equations were given. The particular equation system given by the regression equation allows to calculate characteristic points of coordinates. Examples of four characteristic points were presented in Fig. 1 in the form of black points next to which coordinates such as $\left(f_{S A}, f_{S B}\right)$ or $\left(f_{N S B}, f_{S B}\right)$ were formed. Then, with the use of characteristic points of coordinates the $K\left(f_{n}\right)$ measurement function was formed according to the following Eq. (7):

$$
K\left(f_{n}\right)=\left\{\begin{array}{cc}
f_{n} & \text { for } 0 \leq f_{n} \leq f_{S A}, \\
f_{S B} & \text { for } f_{S A} \leq f_{n} \leq f_{N S B}, \\
f_{n}+A^{*} & \text { for } f_{N S B} \leq f_{n} \leq f_{S C}, \\
C \in\left(f_{S D}, f_{N S D}\right) & \text { for } \quad f_{n}=f_{S C}, \quad(7)
\end{array}\right.
$$

in which $A^{*}=f_{S B}-f_{N S B}$.

According to the form of $K\left(f_{n}\right)$ and according to Eq. (7), it is possible to extract two additional distinctive features i.e. the length of measurement function and the value of area which is included under this function. Given in that way two additional features expand the vector of the basic features of radar signal measurable parameters, such as PW, PRI and RF are a good separation measure in the process of exact identification as far as the copy of a radar is concerned. The way of defining these two additional features and using them in the process of identification of the radar copies of the same type, was presented in the further part of this article.

\section{Fractal of generalized measurement function}

Generalization of the method of radar emission sources identification on the basis of the transformation fractal is defining the generalized measurement function $\hat{K}\left(f_{n}\right)$ going through all particular characteristic points $P_{n}$, in which $n=0,1, \ldots, k_{\text {gr }}$. Figure 2 presents the fractal character of the measurement function received as a result of the transformation of the set of measurement points.

The generalized measurement function $\hat{K}\left(f_{n}\right)$ preserves the character of not decreasing function in a par-

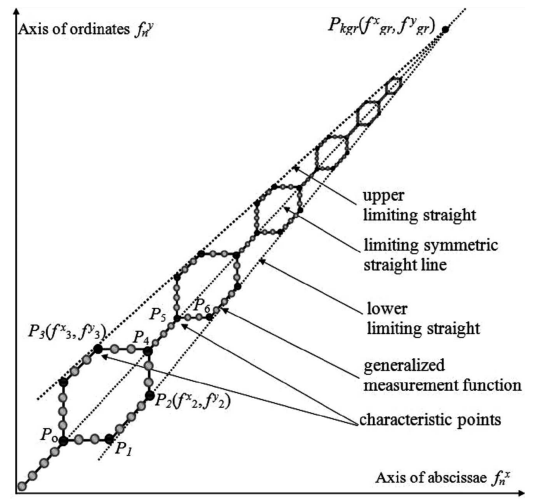

Fig. 2. The fractal character of generalized measurement function going through particular characteristic points. 
ticular bracket $\left\langle f_{n}^{\min }, f_{n}^{\max }\right\rangle$ and out of definite character it shows prediction features. Simultaneously $\hat{K}\left(f_{n}\right)$ is located in an area that is mapped by the lower limiting straight $F_{\mathrm{L}}\left(f_{n}\right)$ and upper limiting straight $F_{\mathrm{U}}\left(f_{n}\right)$ and is symmetric relatively to the symmetrical limiting straight $F_{\mathrm{S}}\left(f_{n}\right)$ according to Eqs. (8-10), in which $f_{2}^{x}, f_{3}^{x}, f_{\mathrm{gr}}^{x}$ are the abscissae of characteristic points and $f_{2}^{y}, f_{3}^{y}, f_{\mathrm{gr}}^{y}$ are the ordinates of characteristic points. Taking into what is above, Fig. 2 presents a fractal character of the generalized measurement function which has the form of a contraction mapping [9]:

$$
\begin{aligned}
& F_{\mathrm{L}}\left(f_{n}\right)=\frac{f_{\mathrm{gr}}^{y}-f_{2}^{y}}{f_{\mathrm{gr}}^{x}-f_{2}^{x}}\left(f_{n}-f_{2}^{x}\right)+f_{2}^{y}, \\
& F_{\mathrm{U}}\left(f_{n}\right)=\frac{f_{\mathrm{gr}}^{y}-f_{3}^{y}}{f_{\mathrm{gr}}^{x}-f_{3}^{x}}\left(f_{n}-f_{3}^{x}\right)+f_{3}^{y}, \\
& F_{\mathrm{S}}\left(f_{n}\right)=\frac{f_{\mathrm{gr}}^{y}}{f_{\mathrm{gr}}^{x}} f_{n} .
\end{aligned}
$$

On the basis of the received depiction, according to Fig. 2, in order to receive universality a generalized form of measurement function was presented. To make it more general, the measurement function $K\left(f_{n}\right)$ in the form of a product degree $k$, given $k+1$ characteristic points, defined by the interpolation Lagrange formula was formulated in accordance with the following form:

$$
\hat{K}\left(f_{n}\right)=a_{k} f_{n}^{k}+a_{k-1} f_{n}^{k-1}+a_{k-2} f_{n}^{k-2} \ldots+a_{0},
$$

in which $a_{k}, a_{k-1}, \ldots, a_{0}$ are characteristic parameters of a generalized measurement function $\hat{K}\left(f_{n}\right)$ given by [10]. The formalized notation of the measurement function $\hat{K}\left(f_{n}\right)$ allows to extract distinctive features through defining the space area under the measurement function and the arc length of the function, which appeared for the SEI process. The feature $\hat{S}$ is the value of the space area of a closed surface expanding from the generalized measurement function $\hat{K}\left(f_{n}\right)$ in the bracket $\left\langle f_{n}^{\min }, f_{n}^{\max }\right\rangle$ respecting Eq. (12):

$$
\begin{aligned}
\hat{S} & =\int_{f_{n}^{\min }}^{f_{n}^{\max }} \hat{K}\left(f_{n}\right) \mathrm{d} f_{n}=\int_{f_{n}^{\min }}^{f_{n}^{\max }}\left(a_{k} f_{n}^{k}+a_{k-1} f_{n}^{k-1}\right. \\
& \left.+a_{k-2} f_{n}^{k-2}+\ldots+a_{0}\right) \mathrm{d} f_{n} .
\end{aligned}
$$

Simultaneously, the arc length of the generalized measurement function $\hat{K}\left(f_{n}\right)$ as the second distinction feature of the radar emission source will be represented through the arc length $\hat{L}$ of the function $\hat{K}\left(f_{n}\right)$ in the brackets $\left\langle f_{n}^{\min }, f_{n}^{\max }\right\rangle$ respecting the following form:

$$
\begin{aligned}
\hat{L} & =\int_{f_{n}^{\min }}^{f_{n}^{\max }} \mathrm{d} f_{n} \sqrt{1+\left(\partial \hat{K}\left(f_{n}\right) / \partial f_{n}\right)^{2}}=\int_{f_{n}^{\min }}^{f_{n}^{\max }} \mathrm{d} f_{n} \\
& \times \sqrt{1+\left(k a_{k} f_{n}^{k-1}+(k-1) a_{k-1} f^{k-2}+\ldots+a_{1}\right)^{2}} .
\end{aligned}
$$

It should be mentioned that the received shape of the measurement function (according to Fig. 1 and Fig. 2) is an individual model of a radar emission source. "An individual model" means "lines on the fingers" of the radar which make a clear identification possible.
4. Identification procedure, analysis results

The results of received measurements' analysis were prepared according to the following schema. At the beginning three copies of the same type of radar were selected for which basic measurable radar signal parameters were filtered. The three copies of radars are shown in Figs. 3-5. Figures 3 and 4 show the graph depicting

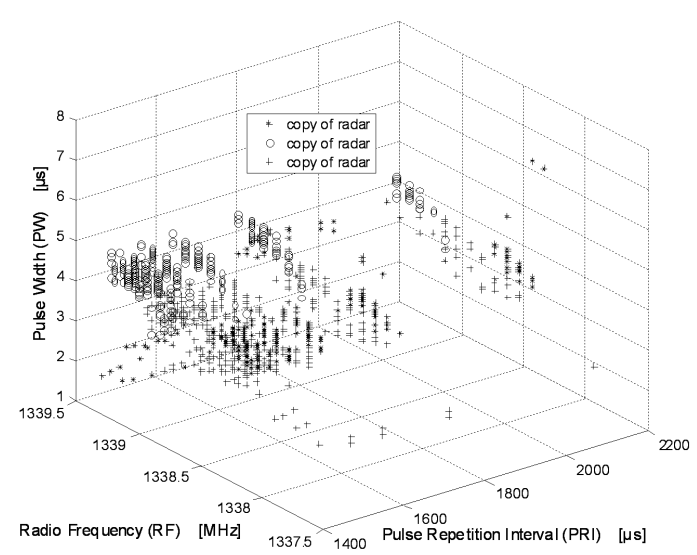

Fig. 3. A graphic depicting of basic measurable parameters of the radar signal, i.e. RF, PRI and PW for three selected copies of the same type of radars.

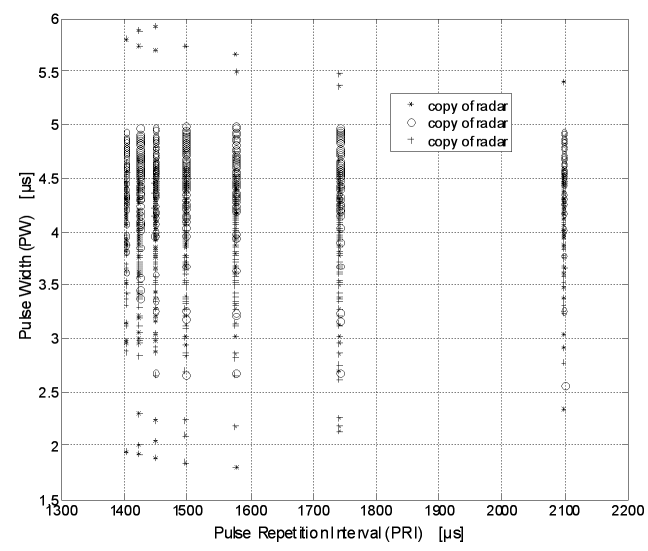

Fig. 4. A graphic depicting of basic measurable parameters of the radar signal i.e. PRI and PW for three selected copies of the same type of radars.

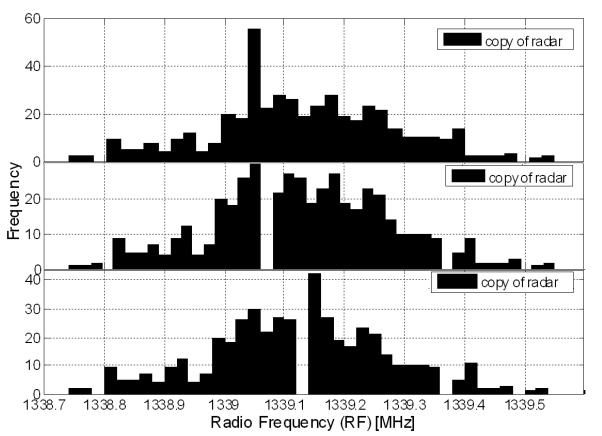

Fig. 5. Radio frequency histogram for three selected copies of the same type of radars. 
basic measurable parameters of the radar signal, i.e. RF, PRI and PW for three selected copies of the same type of radars. In Fig. 5 we can see RF and PRI histograms for three selected copies of the same type of radars.

As can be noticed, primary signal parameters such as PW and especially RF and PRI penetrate each other. For that reason the identification process of the same radar copies with the use of classic methods is almost impossible. The classes of radar models and testing vectors were created on the basis of recorded measurement vectors with the use of the laid-off method. The functional of the conformity mark of the test probe with a particular class was the Mahalanobis $\left(d_{\mathrm{M}}\right)$, Euclid $\left(d_{\mathrm{E}}\right)$ and Hamming $\left(d_{\mathrm{H}}\right)$ distance given by [11]. The nearest neighbour criteria were used as the classification criteria and the correct classification indicator was used in order to assess the quality of the identification process given in [11-15]. To compare the quality of results of the classic identification method with the described SEI method, the following correct identification coefficients (CIC) were defined:

$$
\mathrm{CIC}=L_{\mathrm{g}} / L \text {, }
$$

in which $L_{\mathrm{g}}$ is a number of good classifications, and $L$ is a number of all classifications.

In this experiment about 300 measurement samples were examined. Those were received during the recording of radar signals coming from three copies of the same type of radar. On the basis of recordings test vectors with primary radar signals parameters were defined, i.e. RF, PRI and PW. Test vectors were defined with the use of holdout method, which uses the available data set to divide it into two subsets, the third part for training and the two thirds for testing. Formulae measuring the distance $d_{\mathrm{M}}, d_{\mathrm{E}}$ and $d_{\mathrm{H}}$ and Eq. (14) defined the coefficient $\mathrm{CIC}$, which was estimated as follows: $\mathrm{CIC}=0.169$ for $d_{\mathrm{M}}, \mathrm{CIC}=0.218$ for $d_{\mathrm{E}}$, and CIC $=0.202$ with the use of Hamming distance $\left(d_{\mathrm{H}}\right)$. On the basis of the value of the coefficient above it can be said that there is no possibility to identify radar as far as its copy is concerned in the situation where only primary radar signal parameters are used. What was used further was the expansion of the primary features vector with two additional features i.e. $\hat{S}$ and $\hat{L}$ which were defined as a result of Eq. (12) and Eq. (13), according to the SEI method, which is described in this article. In this case the coefficient CIC $=0.916$ for $d_{\mathrm{M}}$ and CIC $=0.967$ for $d_{\mathrm{H}}$ and $d_{\mathrm{E}}$. On the basis of received values of the correct identification coefficient it can be said that two additional features i.e. $\hat{S}$ and $\hat{L}$ are sufficient measures to identify copies of the same type of emitters.

\section{Conclusions}

Specific identification method of sources of radar emissions presented in this article is based on defining two additional distinctive features which expand the vector of primary parameters of a radar signal. The linear regression method allowed to formalize the notation of measurement function $K\left(f_{n}\right)$ and to extract distinctive fea- tures by mapping the space area under the measurement function and the length of the arc of the resultant function. The analysis of the size of the space area and the estimation of length of its arc introduce additional features to the description of a radar copy (the vector of basic features), which modify the vector of basic measurable parameters of the radar signal. The features above make a piece of distinctive information which is a good separation measurement in order to distinguish exactly the copy of these emission sources. Moreover, as a result of using converted transformations converting sets of measurement points, the transformation fractal of generalized measurement function appeared.

As can be noticed, generalized measurement function $\hat{K}\left(f_{n}\right)$ has the self-similarity feature i.e. a part of it is similar to the whole. It can be said that this function has a fractal character and added that the SEI method described here bases on "fractal features" of the function which is received $\hat{K}\left(f_{n}\right)$. The measurement function which is defined in the form of a contraction mapping will be used in further research in order to optimize the procedure of the specific identification of electromagnetic radiation sources. In further part the authors focus their research on defining the fractal size of received geometrical object $\hat{K}\left(f_{n}\right)$.

\section{References}

[1] K.I. Talbot, P.R. Duley, M.H. Hyatt, Technol. Rev. J. Spring/Summer, 113 ( 2003).

[2] M.W. Liu, J.F. Doherty, in: Proc. 2008 IEEE Sarnoff Symp., Princeton (NJ) 2008, p. 1.

[3] M. Conning, F. Potgieter, in: Proc. 2010 IEEE Radar Conf., Washington 2010, p. 35.

[4] F. Berizzi, G. Bertini, M. Martorella, IEEE Trans. Geosci. Remote Sens. 44, 2361 (2006).

[5] M. German, G.B. Bénié, J.M. Boucher, IEEE Trans. Geosci. Remote Sens. 41, 1765 (2003).

[6] J. Dudczyk, Ph.D. Thesis, Faculties Telecommunication, Univ. of Tech., Warsaw 2004 (in Polish).

[7] L. Kowalski, Statistics, Military Univ. of Technology Press, Warsaw 2001 (in Polish).

[8] Z. Hellwig, Theory of Probability and Mathematical Statistics, PWN, Warsaw 1998 (in Polish).

[9] J. Kudrewicz, Fractals and Chaos, WNT, Warsaw 2007 (in Polish).

[10] R. Leitner, Mathematics for Technical Studies, WNT, Warsaw 1989 (in Polish).

[11] S. Theodoridis, K. Koutroumbas, Pattern Recognition, Academic Press, San Diego 1999.

[12] W. Jakubik, M. Urbanczyk, E. Maciak, T. Pustelny, Bull. Pol. Acad. Sci., Techn. Sci. 56, 133 (2008).

[13] P. Marczynski, A. Szpakowski, C. Tyszkiewicz, T. Pustelny, Acta Phys. Pol. A 122, 847 (2012).

[14] R.O. Duda, P.E. Hart, D.G. Stork, Pattern Classification, Wiley, New York 2000.

[15] K. Fukunaga, Introduction to Statistical Pattern Recognition, Academic Press, New York 1990. 